\title{
MS34-03 | ClPdyn: A Cheap and Reliable ToOl for Molecular Dynamics Studies of Organic Molecules in Condensed Phase
}

Lo Presti, Leonardo (Università degli Studi di Milano, Milano, ITA); Gavezzotti, Angelo (Università degli Studi di Milano, Milano, ITA)

We present CLPdyn, a freely available code intended to perform Molecular Dynamics simulations of organic molecules in condensed phase.[1-3] CLPdyn can handle both continuous phases (liquids, crystals) and finite-size clusters (liquid droplets, nanoparticles), and exploits the thoroughly tested Coulomb-London-Pauli atom-atom intermolecular potential[4,5]. The implementation relies on standard MD algebra, but also includes new algorithms, specifically designed to deal with isolated clusters, to (i) suppress net overall translational and rotational momenta, (ii) handle the evaporation of molecules from the cluster surface, and (iii) measure the amount of residual symmetry from the number and kind of isometries present in the cluster. Application to organic solvents (benzene, chloroform, methanol and pyridine) [2] and crystals spanning very different intermolecular recognition patterns (maleic/succinic anhydrides, alanine/glutamic acid, methylurea, 1,4-cyclohexadiene and methyl-2-amino-5-hydroxybenzoate) [3], shows that CLPdyn reliably reproduces macroscopic thermodynamic quantities, and highlights the effect of the relative strengths of intermolecular forces on rotational correlation times, self-diffusion coefficients and pair distribution functions. Possible applications of CLPdyn to the molecularlevel study of non-equilibrium solution chemistry, including the early stages of crystal nuclei formation, are also discussed.

[1] A. Gavezzotti, CLPdyn, Monte Carlo and Molecular Dynamics modules, Description and user manual, www.angelogavezzotti.it (2018).

[2] A. Gavezzotti, L. Lo Presti, New J. Chem., 2019,43, 2077-2084.

[3] A. Gavezzotti, L. Lo Presti, in preparation

[4] A. Gavezzotti, New J. Chem. 2011, 35, 1360-1368.

[5] A. Gavezzotti and L. Lo Presti, Crystal Growth Des. 2016, 16, 2952-2962. 